\title{
Uniform Oriented Matroids Without the Isotopy Property*
}

\author{
Beat Jaggi, ${ }^{1}$ Peter Mani-Levitska, ${ }^{1}$ Bernd Sturmfels, ${ }^{2}$ and Neil White ${ }^{3}$ \\ 'Mathematisches Institut der Universität Bern, Sidlerstrasse 5 , \\ CH-3012 Bern, Switzerland \\ ${ }^{2}$ Research Institut for Symbolic Computation, Johannes-Kepler Universität, \\ A-4040 Linz, Austria \\ ${ }^{3}$ Department of Mathematics, University of Florida, Gainesville, FL 32611, USA
}

\begin{abstract}
We give an easy general construction for uniform oriented matroids with disconnected realization space. This disproves the longstanding isotopy conjecture for simple line arrangements or order types in the plane.
\end{abstract}

We write $\mathscr{R}(M)$ for the space of all vector realizations $\left(x_{1}, \ldots, x_{n}\right) \in\left(\mathbf{R}^{3}\right)^{n}$ of a rank 3 oriented matroid $M$ on $n$ points. (In other words, $\mathscr{R}(M)$ is the set of $3 \times n$-matrices whose maximal minors have signs given by the alternating map $M:\{1,2, \ldots, n\}^{3} \rightarrow\{-, 0,+\}$.) If $M$ is uniform (i.e., all minors are nonzero) then $\mathscr{R}(\boldsymbol{M})$ is an open subset of $\mathbf{R}^{3 n}$. White's earlier paper [8] gives a nonuniform oriented matroid $M_{W}$ with $\mathscr{R}\left(M_{W}\right)$ disconnected and $n=42$. The aim of the present paper is to present a uniform oriented matroid $\tilde{M}$ with $\mathscr{R}(\tilde{M})$ disconnected. That is, $\tilde{M}$ does not have the isotopy property.

A rank 3 oriented matroid $M$ is said to be constructible if $\left(x_{1}, x_{2}, x_{3}, x_{4}\right)$ is a projective basis and the point $x_{1}$ is incident to at most two lines spanned by $\left\{x_{1}, x_{2}, \ldots, x_{t-1}\right\}$ for $t=5,6, \ldots, n$. Using the configuration $\lambda_{1}=$ $\Omega(17,15,13)\left[\lambda_{0}\right]$ in [4] or a similar modification of White's example [8], we easily get a constructible oriented matroid whose realization space has two connected components. For example, the space $\mathscr{R}\left(\lambda_{1}\right)$ modulo the connected group PGL $\left(\mathbf{R}^{3}\right)$ equals the set of matrices

$$
\left(\begin{array}{rrrrrrrrrrrrrrrcc}
1 & 0 & 0 & 1 & 0 & 1 & 1 & 0 & 1 & 1 & 1 & 1 & 5 & 5 & 0 & 1 & -1 \\
0 & 1 & 0 & 1 & 1 & 0 & -1 & 2 & 2 & 2 & 4 & 6 & 0 & -1 & t & -t & t \\
0 & 0 & 1 & 1 & 1 & 1 & 0 & 1 & 2 & 0 & 1 & 0 & 6 & 5 & 1 & 0 & t-1
\end{array}\right)
$$

\footnotetext{
* The research of Bernd Sturmfels and Neil White was supported in part by the Institute for Mathematics and Its Applications with funds provided by the National Science Foundation.
} 
with $\frac{1}{5}<t<\frac{1}{2}(1-1 / \sqrt{5})$ or $\frac{1}{2}(1+1 / \sqrt{5})<t<\frac{4}{5}$. Hence it suffices to prove the following:

Theorem. Let $M$ be a constructible rank 3 oriented matroid on $n$ points. Then there exists a uniform rank 3 oriented matroid $\dot{M}$ on at most $4(n-3)$ points and a continuous surjective map $\mathscr{R}(\tilde{M}) \rightarrow \mathscr{R}(M)$. Hence $\mathscr{R}(\tilde{M})$ is disconnected whenever $\mathscr{R}(\boldsymbol{M})$ is disconnected.

Proof. We define a sequence $M=: M_{n}, M_{n-1}, M_{n-2}, \ldots, M_{5}, M_{4}=: \tilde{M}$ of oriented matroids and maps between their realization spaces. Let $n \geq t \geq 5$. Then $M_{t-1}$ is constructed from $M_{t}$ as follows. First assume that $x_{i}$ is incident to exactly two lines $x_{i} \vee x_{j}$ and $x_{k} \vee x_{I}$ with $1 \leq i, j, k, l<t$. Using the notation of Billera and Munson [1], we let $M_{i}^{\prime}$ be the oriented matroid obtained from $M_{t}$ by the four successive principal extensions

$$
\begin{array}{ll}
x_{i, 1}:=\left[x_{i}^{+}, x_{i}^{+}, x_{k}^{+}\right], & x_{i, 2}:=\left[x_{i}^{+}, x_{i}^{+}, x_{k}^{-}\right], \quad x_{i, 3}:=\left[x_{i}^{+}, x_{i}^{-}, x_{k}^{-}\right], \\
& x_{i, 4}:=\left[x_{i}^{+}, x_{i}^{-}, x_{k}^{+}\right] .
\end{array}
$$

These extensions can be carried out for every vector realization of $\boldsymbol{M}_{t}$ by setting

$$
\begin{array}{ll}
x_{t, 1}:=x_{t}+\varepsilon_{1} x_{i}+\varepsilon_{2} x_{k}, & x_{t, 2}:=x_{t}+\varepsilon_{3} x_{i}-\varepsilon_{4} x_{k}, \\
x_{t, 3}:=x_{t}-\varepsilon_{5} x_{t}-\varepsilon_{6} x_{k}, & x_{i, 4}:=x_{i}-\varepsilon_{7} x_{i}+\varepsilon_{8} x_{k}
\end{array}
$$

where $1 \gg \varepsilon_{1} \gg \varepsilon_{2} \gg \cdots \gg \varepsilon_{8}>0$. This implies that the deletion map $\Pi: \mathscr{R}\left(M_{t}^{\prime}\right) \rightarrow$ $\mathscr{R}\left(M_{t}\right)$ is surjective. Geometrically speaking, in every affine realization of $M_{\prime}^{\prime}$, the intersection point $x_{t}$ is "caught" in the quadrangle $\left(x_{t, 1}, x_{t, 2}, x_{t, 3}, x_{t, 4}\right)$. Define $M_{t-1}:=M_{t}^{\prime} \backslash x_{t}$ by deletion of that point, and let $\pi: \mathscr{R}\left(M_{t}^{\prime}\right) \rightarrow \mathscr{R}\left(M_{t-1}\right)$ denote the corresponding map. (See Fig. 1.)

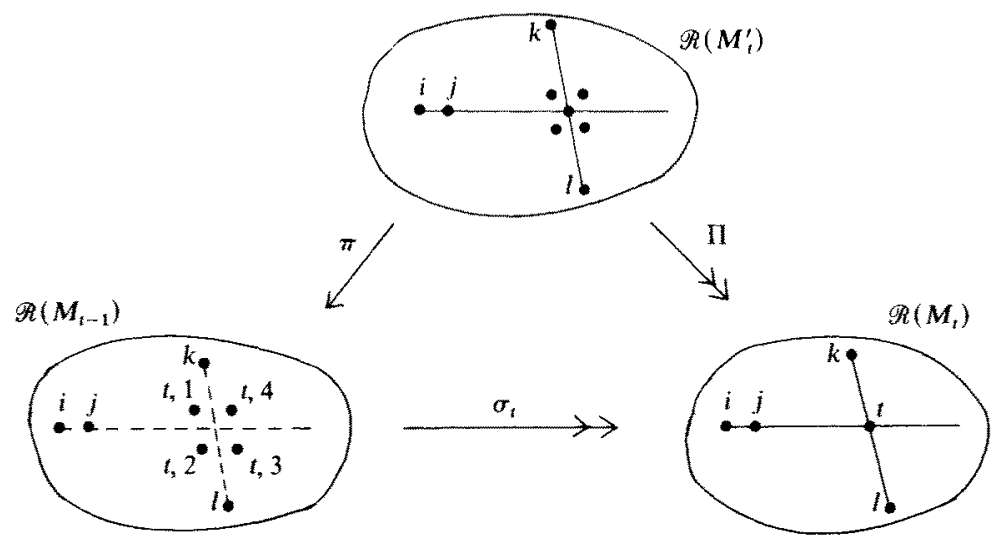

Fig. 1. Illustration of the oriented matroids $M_{t}, M_{i}^{\prime}$, and $M_{t-1}$. 
Next consider an arbitrary realization $X:=\left(x_{1}, \ldots, x_{1}, x_{t, 1}, x_{1,2}, x_{1,3}, x_{1,4}\right.$, $\left.x_{r+1,1}, \ldots, x_{n, 4}\right)$ of $M_{1}$, As a consequence of the principal extension construction used in (1), $x_{k} \vee x_{k}$ and $x_{k} \vee x_{1}$ are the only lines spanned by $\left\{x_{1}, \ldots, x_{1}\right.$, , $\left.x_{1+1,1} \ldots, x_{n, 4}\right)$ which intersect the quadrangle $\left(x_{t, 1}, x_{t, 2}, x_{t, 3}, x_{t, 4}\right)$. For any other such line the intersection point $x_{1}:=\left(x_{1} \vee x_{1}\right) \wedge\left(x_{k} \vee x_{1}\right)$ is on the same side as $x_{1,1}, \ldots, x_{1,4}$. Therefore $\sigma_{1}(X):=\left(x_{1}, \ldots, x_{1}, x_{1+1,1}, \ldots, x_{n, 4}\right) \in \mathscr{A}\left(M_{1}\right)$.

Hence we have a well-defined continuous map $\sigma_{1}: A\left(M_{1},\right) \rightarrow \mathscr{A}\left(M_{1}\right), X \mapsto$ $\sigma_{1}(X)$. Moreover, $\sigma_{b}$ is surjective because $I I=\sigma_{1} \circ \pi$ is surjective.

It remains to define $M_{1}$, and $\sigma$, when $x_{1}$ is incident to less than two lines in

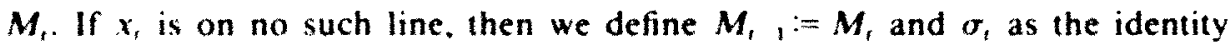
map. Finally, suppose that $x_{t}$ is on only one line $x_{1}, x_{,}, 1 \leq i, j<t$. In that case we replace (1) by setting $x_{i, 1}:=\left[x_{i}^{*}, x_{k}^{*}\right], x_{t, 2}:=\left[x_{i}^{*}, x_{i}^{*}, x_{k}\right], x_{i, 3}:=\left[x_{i}^{*}, x_{1}, x_{k}\right]$ for some $x_{k}, x_{2} \vee x_{n}$, and in the definition of the map $\sigma_{r}$ we set $x_{f}:=\left(x, \vee x_{k}\right) \wedge\left(x_{k} \vee x_{k, 1}\right)$.

Iterating these constructions resolves all previous dependencies, and we obtain a uniform oriented matroid $\bar{M}:=M_{4}$ on $4(n-3)$ or fewer points. Moreover, we have a continuous surjection $\sigma:=\sigma_{n}=\sigma_{n}, \cdots, \sigma_{s}$ from $\mathcal{A}(\tilde{M})$ onto A $(M)$.

Remarks. (a) Using a fairly straightforward procedure for doubling oriented matroids, we get the following corollary: Given any integer $C$, there exists a uniform rank 3 oriented matroid $\dot{M}_{6}$ with $4(n-3) C$ points such that $\mathcal{A}\left(\dot{M}_{6}\right)$ has at least $2^{\prime}$ connected components.

(b) After this paper had been accepted for publication we learned that the isotopy problem for uniform oriented matroids had been solved independently in 1985 by Mnev [9]. This result is pan of a very general theory of configuration spaces developed by a group of Soviet topologists [10]. For further details see also [11].

(c) The construction presented here is an essentially simplified version of a construction originally found by the first two authors [4].

\section{References}

1. L. J. Billera and B. S. Munson, Triangulations of oriented matroids and convex polytopes, SIAM J. Algebraic Discrele Methods 5 (1984). $515-525$.

2. 1. Bokowski and B. Sturmfels, On the coordinatization of oriented matroids, Discrete Comput. Geom. 1 (1986), 293 -306.

3. R. Cordovil, Oriented matroids of rank three and arrangements of pseudolines, Ann. Discrete Math. $17(1983), 219-223$.

4. B. laggi and P. Mani-Levitska, A simple arrangement of lines without the isotopy property. Manuscript, Bern, January 1988.

5. 1. E. Goodman and R. Pollack. Upper bounds for configurations and polytopes in $R^{d}$, Discrete Comput. Ceom. 1 (1986), 219-227.

6. J. Richter and B. Sturmfels. On the topology and geometric construction of oriented matroids and convex polytopes, submitted.

7. G. Ringel, Teilungen der Ebene durch Geraden oder topologische Geraden, Math. Z. 64 (1956). 79-102. 
8. N. White, A nonuniform matroid which violates the isotopy conjecture, Discrete Comput. Geom. $4(1989), 1-2$.

9. N. E. Mnev, The universality theorems on the classification problem of configuration varieties and convex polytope varieties, in Topology and Geometry-Rohlin Seminar (O. Y. Viro, ed.), Lecture Notes in Mathematics, Vol, 1346, Springer-Verlag, Berlin, 1988.

10. A. M. Vershik, Topology of the convex polytopes' manifold, the manifold of the projective configurations of a given combinatorial type and representations of lattices, in Topology and Geometry-Rohlin Seminar (O. Y. Viro, ed.), Lecture Notes in Mathematics, Vol. 1346, SpringerVerlag, Berlin, 1988.

11. B. Sturmfels, Computational Synthetic Geometry, Lecture Notes in Mathematics, Springer-Verlag, Berlin, to appear.

Received February 11, 1988, and June 11, 1988, and in revised form July 15, 1988. 